\title{
¿Cómo se puede mejorar la competitividad de las cooperativas agroalimentarias?
}

\author{
Natalia Lajara-Camilleri \\ Ricardo Server-Izquierdo
}

RESUMEN: El entorno de alta competitividad que existe en los mercados agroalimentarios ha incrementado notablemente la presión sobre todos los agentes que operan en ellos. En el caso de las cooperativas, debido posiblemente a su reducida dimensión empresarial, esta cuestión reviste especial importancia puesto que aboca a estas entidades a adoptar cambios que mejoren su competitividad y aseguren la supervivencia. Existe un buen número de trabajos que analizan el papel de distintos aspectos de gestión sobre la competitividad de las empresas en general, sin embargo no se ha abordado el grado de importancia relativa de dichos elementos. El objetivo de este trabajo es establecer una jerarquía en una serie de aspectos de gestión empresarial que inciden en el éxito y competitividad de las cooperativas agroalimentarias. Para ello se ha realizado una consulta a expertos mediante el método Delphi a fin de obtener un orden de importancia percibida por parte del sector. Los resultados obtenidos señalan a la orientación al mercado y a la innovación como los elementos más destacados por los expertos de cara a mejorar la competitividad actual de las cooperativas agroalimentarias españolas.

PALABRAS CLAVE: Cooperativas agroalimentarias, competitividad, formación, innovación, internacionalización, orientación al mercado, Delphi.

CLAVES ECONLIT: M10, Q13, M21.

Cómo citar este artículo / How to cite this article: LAJARA-CAMILLERI, N. \& SERVER-IZQUIERDO, R. (2017): "¿Cómo se puede mejorar la competitividad de las cooperativas agroalimentarias?", CIRIEC-España, Revista de Economía Pública, Social y Cooperativa, 90, 103-121.

Correspondencia: Natalia Lajara-Camilleri, Ayudante Doctor, y Ricardo Server-Izquierdo, Catedrático de Universidad. CEGEA - Centro de Investigación en Gestión de Empresas, Universitat Politècnica de València. E-mail de contacto: nalade@cegea.upv.es. 


\section{EXPANDED ABSTRACT}

\section{How can the competitiveness of agro-food cooperatives be improved?}

\section{Objectives}

The environment of high competitiveness that exists in agro-food markets has significantly increased the pressure on all the agents that operate in them. In the case of cooperatives, possibly due to their small business size, this issue is of particular importance as it encourages these entities to adopt changes that pursue to improve their competitiveness, ensuring their survival. There is a good number of papers that partially analyze the role of different aspects of management on the competitiveness of cooperatives (Juliá et al., 2012; Arcas, 2002; Moyano et al., 2008; among others). In previous work, we have selected a set of six elements of management that are crucial to the competitiveness of agrifood cooperatives, they are known as DIDIFO, which stands for Dimension (size) - Internationalization - Diversification - Innovation - Formation (training) - (market) Orientation (Lajara \& Server, 2016). The contribution to a better performance in companies is undoubtable but the degree of relative importance among these elements has not yet been addressed. What is more relevant for a cooperative to increase its competitiveness, to improve its size or to diversify its portfolio? The objective of this work is to establish a hierarchy of aspects in business management that affect the success and competitiveness of agro-food cooperatives.

\section{Method}

This question could only be answered using a qualitative approach. For this work, an expert consultation has been carried out using the Delphi method in order to obtain an order of importance as perceived by the sector. A number of experts were selected and grouped according to their relationship to cooperatives: representative associations, local government, universities and cooperative staff. They were asked to order the factors as well as to include any other aspect they found interesting related to the topic. The questionnaire was sent by e-mail and two rounds of consultation were needed to achieve a degree of consensus and stability.

\section{Results}

28 valid answers were collected after the second round. In this phase, either consensus or stability was achieved in all the questions. Results permit the formulation of a proposal of hierarchy even if experts could not agree on a unique order. Both market orientation and innovation have been pointed 
as the most outstanding elements by experts in order to improve the current competitiveness of Spanish agro-food cooperatives. Diversification occupies the second place in the ranking, followed by size, internationalization and training. There is consensus in the positions of market orientation and diversification and also in the rest of the factors, there is a level of stability that shows that answers would not change in following rounds.

\section{Conclusions}

Market orientation and innovation are elements that have been proposed by consensus as the most relevant when it comes to encouraging competitiveness. This result is consistent with the current stream of management in which the need to satisfy not only the customer but the market is high. In addition, in a sector such as agro-food in which commodities abound, it is vital to seek differentiation and one of the most effective ways to achieve this is innovation.

The following factors with respect to importance are dimension (size), internationalization and training. Particularly noteworthy is the latter aspect, training, as it highlights one of the problems that traditionally has been attributed to the sector which is the lack of professionalization. We can say that the fact that it has been categorized as the least important factor within the proposed ones denotes in some way that this mentality in the sector still persists.

In future research it would be interesting to analyze whether there are significant differences between the different groups of experts who have participated in the study, that is, if managers of cooperatives have opinions that are statistically different from those provided by the researchers or the staff of the Administration. In addition, it could be explored whether two types of factors could be established: a group of cross-cutting elements that enhance other aspects (eg training) and a more specific one, integrated by innovation or internationalization.

\section{Contribution}

This work helps to increase the existing evidence about management in cooperatives. This kind of business can be a way of empowering employees in new founded-companies that are coming up in this revolution post-crisis. But to give them the opportunity of surviving and succeed, we still need to find strategies to update the way they are managed. Results of this paper show that effort has to be placed mainly in market orientation and innovation but keeping in mind that there is still a problem of professionalization in the sector.

KEYWORDS: Agrifood cooperatives, competitiveness, education, innovation, internacionalization, market orientation, delphi. 


\section{1.- Introducción y objetivos}

La sociedad cooperativa es un tipo particular de empresa donde la participación activa y efectiva en el proceso de producción y/o comercialización es el hecho que posibilita y legitima la capacidad para tomar decisiones de forma democrática acerca de su gestión, fundamentalmente a través de la definición de objetivos (García-Gutiérrez, 1988). Se trata de una forma de empresa social, con un propósito que beneficia a la sociedad y un proceso de toma de decisiones democrático y participativo (Monzón y Herrero, 2016).

Resulta indiscutible el rol que desempeñan las cooperativas agroalimentarias tanto de cara a sus socios, mejorando el poder de negociación frente a terceros y a través de los servicios que prestan, como al conjunto de la sociedad al abastecer de alimentos saludables y contribuir al desarrollo y la cohesión social del medio rural (Arcas et al., 2013).

\section{Figura 1. Funciones de las cooperativas agroalimentarias}

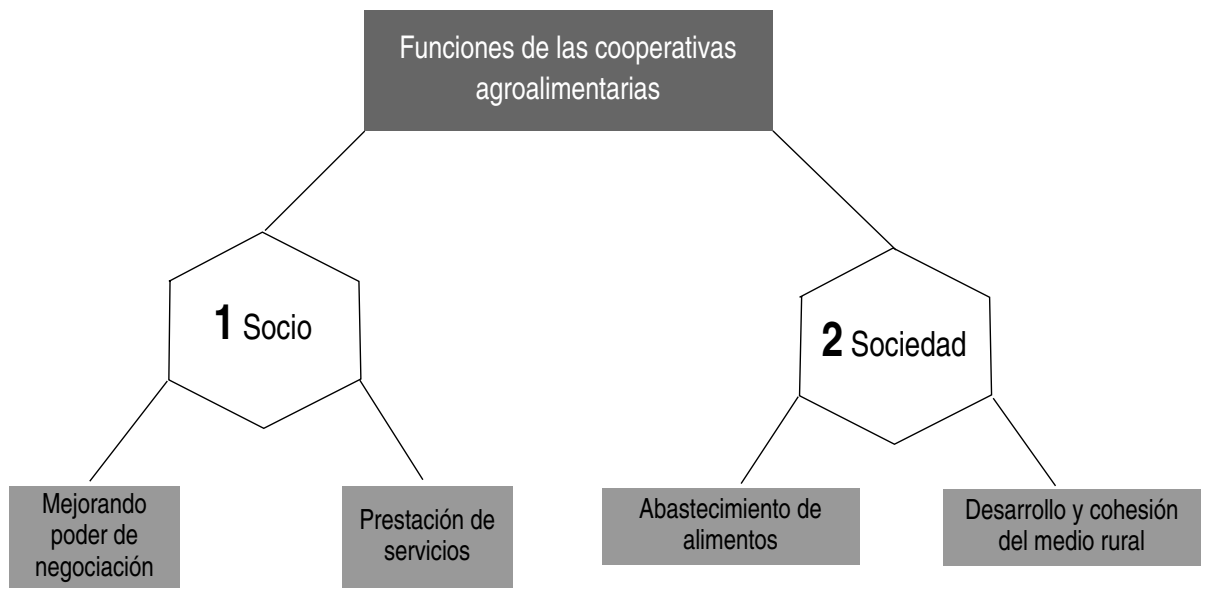

FUENTE: Arcas et al., 2013.

Las asimetrías de la distribución alimentaria se han exacerbado en la última década y hacen necesario potenciar los instrumentos de concentración de la producción frente a empresas que demandan elevados volúmenes y que prácticamente imponen las condiciones de las ventas. 
Actualmente en España existen 3.397 cooperativas agroalimentarias (OSCAE, 2013) cuyo cometido fundamental es aglutinar la oferta y ejercer de vehículo transmisor de las demandas del mercado. En conjunto proporcionan empleo directo a 97.944 trabajadores y presentan una facturación global de 25.696 millones de euros, lo que representa aproximadamente un $60 \%$ de la Producción Final Agraria (MAGRAMA, 2014).

Coincidiendo con Juliá et al. (2013), cabe recordar que la agricultura actual se enfrenta a tres retos fundamentales: la globalización e inevitable liberalización de los mercados, los cambios en la demanda y la reforma de la PAC. La/s estrategia/s que adopten las cooperativas respecto a ellos determinará su supervivencia en el medio y largo plazo. Bretos y Marcuello (2017) profundizan en los retos que supone la globalización para las cooperativas y señalan tres elementos como clave: viabilidad, desarrollo local e internacionalización. Tal como afirman Alonso y Santa Cruz (2016), el cooperativismo actual necesita reformularse como un modelo de empresa diferente, con objetivos sociales de creación de empleo y democratización social, manteniendo sus señas de identidad, pero que al mismo tiempo le permita crecer.

Por esta razón, es adecuado estudiar los aspectos de gestión empresarial que inciden en la ventaja competitiva de las cooperativas, a fin de dotar al sector de la información necesaria para poder establecer justificadamente las medidas que permitan a las cooperativas agroalimentarias mantenerse y crecer dentro del mercado.

El objetivo de este trabajo es establecer un ranking de importancia de los elementos de gestión empresarial que la literatura señala como clave en el éxito y competitividad de las cooperativas agroalimentarias. Para ello se utilizará una consulta a expertos a fin de obtener la jerarquía percibida por parte del sector.

\section{2.- Marco conceptual}

La competitividad empresarial es una cuestión que ha sido ampliamente analizada en la literatura académica; los trabajos de Porter sobre las cinco fuerzas competitivas o la teoría de recursos y capacidades son la base de numerosos estudios empíricos que se han aplicado principalmente en empresas capitalistas. Aunque es cierto que las cooperativas pueden tomar como referencia sus conclusiones, no se puede dejar de observar que la idiosincrasia de este tipo de empresas de la economía social justifica que se lleve a cabo un estudio específico sobre su aplicación. 
En España la competitividad en cooperativas ha sido abordada por un cierto número de autores (Juliá et al., 2012; Arcas, 2002; Moyano et al., 2008; entre otros). A través de estudios sectoriales 0 abordando elementos concretos de la gestión se ha analizado la incidencia de diversos factores en la competitividad de las cooperativas agroalimentarias.

De la literatura existente podemos concluir que existen seis factores clave que afectan a la competitividad de las cooperativas agroalimentarias: dimensión (Melián y Millán, 2007; Nilsson et al., 2009; Juliá et al., 2012; Arcas-Lario et al., 2014; Meliá y Martínez, 2015, entre otros), internacionalización (Fayos et al., 2011; Juliá et al., 2012), innovación (Triguero-Cano y Cuerva, 2011; Juliá et al., 2012; Guzmán et al., 2013; Saiz y Mendoza, 2015, entre otros), formación (Campos-Climent et al., 2012; Campos-Climent y Chaves-Ávila, 2012; entre otros), diversificación (Juliá et al., 2012 y Campos-Climent y Chaves-Ávila, 2012; entre otros) y orientación al mercado (Hernández-Espallardo y Arcas-Lario, 2003; entre otros).

La dimensión es una cuestión sobre la que aparentemente existe consenso acerca de la importancia que reviste a nivel de gestión empresarial. Es necesario alcanzar un tamaño mínimo que permita acceder a economías de escala, a directivos profesionales y ser capaz de afrontar el volumen de negocio que imponen los actuales interlocutores en el mercado agroalimentario (Campos-Climent et al., 2012).

La internacionalización se define como la ampliación de las actividades comerciales de la empresa más allá del territorio nacional en el que se ubica. Se trata de un proceso que puede ser gradual, comenzando con actividades puntuales de exportación y creando en último término multinacionales. Tal como afirman Moral-Pajares et al. (2015), la exportación conduce a las empresas a una mayor eficiencia al permitirles competir en entornos globales.

El interés en relación con las cooperativas agroalimentarias radica en la importancia que tradicionalmente ha tenido la actividad exportadora en la facturación de muchas de las cooperativas españolas, especialmente en el sector hortofrutícola. Gallego y Lamanthe (2011) sostienen que la orientación hacia la exportación ha estimulado el desarrollo de un know-how productivo-comercial que ha sido la base para la mejora continua en los mercados.

No obstante, hoy en día el reto que supone la globalización empuja inevitablemente a la apertura de nuevos mercados, lo que implica atravesar fronteras y descubrir potenciales consumidores. Las cooperativas deben estar preparadas para hacer frente a los requerimientos que supone este reto y la competencia que van a encontrar.

En relación a la formación, el estudio de Arcas et al. (2013) llevado a cabo sobre una muestra de 277 socios de cooperativas agroalimentarias de la Región de Murcia pone de manifiesto el escaso nivel de educación formal que existe actualmente en este sector. El $40 \%$ de los encuestados no poseían estudios mientras que el $42 \%$ contaban con estudios primarios, el resto contaba con Bachiller ( $9 \%)$, 
formación profesional (6\%) o estudios universitarios (2\%). Las cooperativas son una herramienta eficaz para paliar estas carencias por un doble motivo: por una parte muestran una preocupación manifiesta en la formación y cuentan con reservas específicas para ello y por otra parte, en la medida que los trabajadores de las cooperativas sean profesionales debidamente formados, éstos pueden ayudar con su desempeño a nivel de planificación, asesoramiento y comercialización.

La formación es un elemento imprescindible en la consecución de ventajas competitivas (Rodríguez y Mozas, 2003). Tal como afirma Montegut (2006), es un aspecto clave para que las cooperativas afronten con éxito las presiones competitivas a las que son sometidas. Es un hecho aceptado que cada vez son más necesarios profesionales suficientemente formados en sus áreas, que conozcan no sólo las mejoras técnicas de gestión, producción, comercialización, etc. sino que a su vez sean conscientes de las especificidades del sector cooperativo agrario (Encinas et al., 2011; Melián y Campos, 2009). De forma similar a lo que sucede con la dimensión empresarial, la formación posibilita y potencia el resto de factores competitivos.

La diversificación es la introducción de nuevos productos, servicios o actividades de la cooperativa. Esta ampliación de la oferta puede entenderse tanto como el desarrollo de productos adicionales asociados a los que se venían produciendo, la búsqueda de nuevos mercados/clientes o bien el establecimiento de acuerdos o fórmulas de intercooperación para realizar comercialización en común. Es un concepto unido por tanto al de innovación.

La diversificación conlleva una reducción de la concentración del riesgo ya que se reduce la dependencia frente a determinados mercados, clientes, etc. Desde un punto de vista de gestión resulta una oportunidad para mejorar la rentabilidad. Estratégicamente mejora la posición competitiva de una cooperativa al ser capaz de ofrecer a sus clientes una gama más completa de productos o servicios. Por ello, se hace necesario establecer los mecanismos necesarios para fomentar la innovación tanto a nivel de producto o servicios como en el plano de procesos (Rubio-Bañón y Aragón-Sánchez, 2002).

La orientación al mercado es la vinculación con el consumidor final en la medida en que la cooperativa sea capaz de percibir o incluso adelantarse a las necesidades del cliente y satisfacerlas de manera rentable. Se trata de una opción estratégica para la cooperativa (Benos et al., 2015) y junto con la orientación a la marca, determina la aproximación de marketing de la cooperativa (Urde et al., 2013).

Arcas (2002) subraya la dificultad que encuentran las cooperativas en este sentido, ya que la preocupación por la comercialización del producto que entregan sus socios y su ubicación al inicio de la cadena alimentaria propicia en las cooperativas una orientación más próxima a la producción que al mercado. Kyriakopoulos (1998) analiza con detenimiento las ventajas que supone la orientación al mercado para las cooperativas y señala cómo pueden éstas reorientarse hacia el mercado. 
Cambra y Fuster (2005) señalan la necesidad de adoptar una estrategia de orientación al mercado a fin de ser capaces de "percibir las amenazas del entorno y ser rápidas a la hora de detectar las necesidades reales y futuras de sus clientes para poder satisfacerlas". Lajara-Camilleri y Server (2016) abordan esta cuestión, poniendo de manifiesto no sólo la importancia de la orientación al mercado en la competitividad sino la relación que existe entre orientación al mercado e innovación en cooperativas agroalimentarias.

La innovación es una cuestión de actualidad en las empresas y su entorno. La sociedad del cambio empuja a las organizaciones a someterse a una continua adaptación, además la creciente liberalización del comercio recrudece las condiciones de competencia y hace necesario incrementar el valor añadido del producto. Para todo ello es necesaria la innovación, ya sea de producto, de proceso, de organización o de comercialización. Además, hay que tener en cuenta la importancia creciente de las llamadas innovaciones sociales, concepto aún en desarrollo pero que introduce explícitamente a la sociedad (Hernandez-Ascanio et al., 2016), sobre las que autores como Vézina et al. (2017) sostienen que las fórmulas de economía social -entre ellas las cooperativas- que cuentan con suficiente madurez y experiencia tienen una capacidad única para llevar a cabo innovaciones sociales, debido a su carácter colectivo y a sus procesos democráticos y deliberativos. La innovación es, por tanto, un factor de competitividad en el entorno empresarial (Hernández-Espallardo et al., 2011; Tamayo et al., 2015; Peraza et al., 2016).

En cooperativas su relevancia ha sido puesta de manifiesto en los trabajos de Fernández de Bobadilla y Velasco (2008), Triguero-Cano y Cuerva (2011) y Juliá-Igual et al. (2012).

\section{3.- Metodología}

Para la realización de este estudio se ha recurrido a la metodología Delphi de consulta a expertos. Se trata de una técnica que permite recabar información amplia de un panel y llevar a cabo jerarquizaciones. Cuenta con dos ventajas fundamentales: por una parte, al contar con la opinión de un grupo de expertos, el consenso al que se llegue, el resultado que se obtenga, será la visión que ofrezca mayor confianza. Además permite superar limitaciones de otras técnicas cualitativas como las entrevistas en grupo o grupos focales ya que al no existir interacción directa entre los participantes, se limita la influencia de los sujetos dominantes, el ruido semántico y la presión hacia la conformidad (Martínez Piñeiro, 2003).

El punto de partida para el desarrollo de la técnica Delphi es la identificación de los objetivos a estudiar, mediante la formulación de una serie de hipótesis de trabajo, que deben plantear un pro- 
blema o conjunto de problemas susceptibles de ser tratados por medio de esta metodología (Delbecq et al., 1989).

La idea que subyace en los planteamientos del presente estudio tiene su origen en la necesidad de armonizar posturas entre las partes implicadas, en lo que respecta a la incidencia de los aspectos de gestión propuestos en la competitividad de las cooperativas. Es decir, se trata de llegar a obtener una jerarquización consensuada de los factores que, a ojos de los expertos, resultan claves para mejorar la competitividad de las cooperativas agroalimentarias españolas.

Así pues, la opinión del grupo de expertos, procesada mediante la técnica Delphi, permite aunar las diferentes perspectivas de todos los participantes, con perfiles distintos, sobre la materia en cuestión. Cada experto consultado debe pronunciarse sobre la importancia que conceden a cada aspecto de gestión.

El fundamento de este método reside en que el juicio integrado de un grupo de personas dotadas de un elevado nivel de capacidad crítica puede realizar una aproximación con mayor fidelidad que cualquier estimación individual (Juliá y Gallego, 2003).

Para ello se llevan a cabo tantas rondas de consulta como sean necesarias hasta alcanzar bien el consenso en el grupo o una estabilidad en la respuesta que permita afirmar que sucesivas rondas no obtendrían resultados diferentes.

Los aspectos que van a caracterizar cada ronda son tres: por un lado, la opinión de los expertos; por otro, el consenso, entendido como el nivel de acuerdo que existe entre las respuestas dadas por todos los expertos; y finalmente la estabilidad, entendida como la variación en la opinión de los expertos en las sucesivas rondas de envío del cuestionario.

Así, con la finalidad de conocer la posición del grupo de expertos ante cada una de las preguntas se determina tanto la mediana $(\mathrm{m})$ como la media $(\mu)$ de la muestra en cada una de ellas. Además, para determinar el consenso se ha establecido adoptar como criterio que el intervalo intercuartílico (IIC) sea igual o inferior a 1. El intervalo intercuartílico se define como el rango entre el primer y el tercer cuartil y cuenta con la resistencia de la mediana, ya que los valores extremos no tienen una incidencia grave en él (Martínez Piñeiro, 2003).

$$
\text { IIC }=Q_{3}-Q_{1}
$$

A fin de evaluar la estabilidad de las respuestas se utiliza la variación del rango intercuartílico relativo (RIR), es decir el intervalo intercuartílico de cada ronda dividido por su mediana. Cuando la variación se encuentre entre -0,5 y 0,5 se entiende que existe un nivel satisfactorio de estabilidad en la respuesta del grupo y que no es probable que cambie en rondas sucesivas. En ese caso, exista o no consenso, la respuesta se tomará como definitiva. Cuando la variación supera los límites anteriores, 
se ha producido un cambio importante en las opiniones del grupo, dando a entender que las posturas no son inamovibles.

$$
\begin{gathered}
\mathrm{RIR}=\left(\mathrm{Q}_{3}-\mathrm{Q}_{1}\right) / \mathrm{Md} \\
\text { Variación } \mathrm{RIR}=\mathrm{RIR} \mathrm{R}_{1}-\mathrm{RIR} 2
\end{gathered}
$$

\section{4.- Desarrollo del estudio}

Para el estudio se determinaron en primer lugar los grupos de expertos que integran el panel con el fin de obtener información de todos los actores interesados y conocedores del sector. Los grupos definidos son entidades representativas de las cooperativas agrarias en la Comunidad Valenciana, Administración, Centros de formación y/o Universidades y Personal de cooperativas.

De este modo, quedaban cubiertos los diferentes colectivos que podían realizar aportaciones útiles al ámbito de este estudio.

La descripción y número de expertos que participaron en las dos fases del estudio es el siguiente:

- Entidades representativas de las cooperativas agrarias en la Comunidad Valenciana: 2 expertos, procedentes de sindicatos agrarios o federaciones de cooperativas, con dilatada experiencia en el sector y ocupando cargos de gestión.

- Administración: 4 expertos relacionados con el ámbito cooperativo desde distintas posiciones (administración local en materia de agricultura, alimentación, ganadería y pesca, registro, comercio exterior, etc.)

- Centros de formación y/o Universidades: 4 investigadores en materia de economía social

- Personal de cooperativas: 18 gerentes de cooperativas.

El cuestionario fue remitido vía correo electrónico a todos los expertos debido a la rapidez y comodidad que supone tanto a la hora de cumplimentarlo como en el envío en sí. Las respuestas se recibieron por la misma vía.

Para el tratamiento y análisis estadístico de la información se ha utilizado tanto el programa Excel de Microsoft Office como el SPSS v16. 


\section{5.- Resultados}

\subsection{Primera ronda}

La selección inicial de expertos estaba formada por 30 miembros, escogidos en función de su vinculación desde diferentes ámbitos al sector del cooperativismo agrario. De esta muestra inicial se recibieron 28 cuestionarios válidos, cuyo contenido integra los resultados de la primera ronda.

Las respuestas obtenidas están comprendidos entre 1 y 6 puesto que se refieren a la jerarquización de los elementos propuestos. En la primera ronda el criterio de estabilidad equivale al de consenso, es decir, se considera que en el caso de existir acuerdo, el resultado será estable.

Cabe señalar que las preguntas abiertas no han sido tratadas estadísticamente aunque se incluyen las respuestas obtenidas en el correspondiente apartado.

En un primer análisis, las opiniones de los expertos son tratadas de forma conjunta para analizar la opinión del grupo y determinar si existe consenso respecto a los distintos ítems que integran las preguntas del cuestionario.

En la primera consulta realizada, tan sólo se alcanza el consenso en relación a la orientación al mercado, aspecto que el $62 \%$ de los expertos coincide en señalar como el más importante en la mejora de la competitividad de las cooperativas agroalimentarias. En el resto de casos existe divergencia de opiniones aunque se llega a obtener una jerarquización de los aspectos en base a las opiniones vertidas por los expertos.

Los temas que suscitan mayores divergencias entre los expertos en la primera ronda son, por orden:

- Diversificación. Los datos obtenidos son singulares. Por una parte el 40\% de los expertos coinciden en situar este factor como el segundo en importancia de entre los propuestos, no obstante, las opiniones del grupo en conjunto son las que presentan las diferencias más importantes, con un intervalo intercuartílico de 4.

- Innovación. El 36\% de los expertos la señala como el elemento más importante de la gestión de las cooperativas agroalimentarias a la hora de mejorar la competitividad, no obstante su intervalo intercuartílico es de 3. 
- Formación. Es otro elemento de controversia. Pese a que el 32\% de los expertos opinan que es el quinto aspecto más importante en la gestión de cara a la mejora de la competitividad, el intervalo intercuartílico se sitúa -al igual que en el caso de la innovación- en 3.

Los resultados sobre la distribución de los ítems de las preguntas en función de que se haya logrado el consenso en la primera ronda o no, se muestran en la Figura 2.

\section{Figura 2. Consenso en las respuestas en la primera ronda (grado de coincidencia por parte de los expertos en la respuesta más frecuente)}

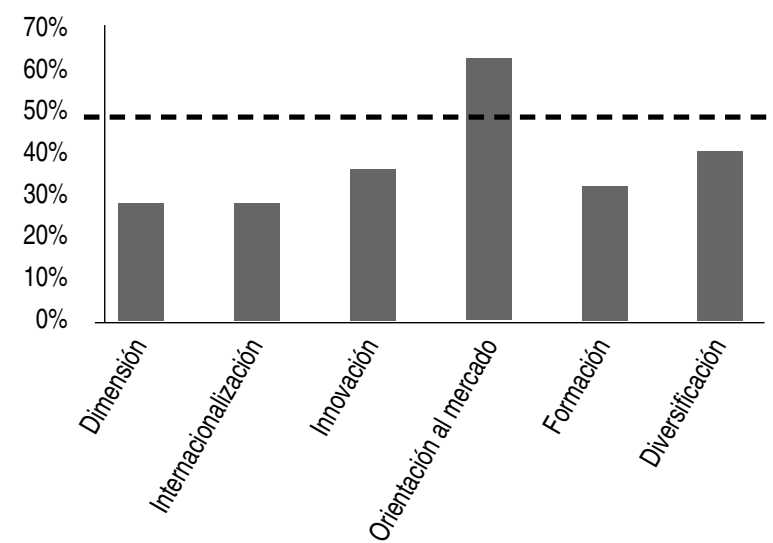

\subsection{Segunda ronda}

Una vez finalizada la segunda ronda de consulta a expertos, los cuestionarios son analizados a fin de obtener los resultados generales correspondientes al grupo. En la segunda ronda los expertos han tenido ocasión de conocer la respuesta estadística del grupo, compararla con la que ellos proporcionaron en su momento y modificarla si lo estiman oportuno. Por esta razón al análisis de consenso establecido inicialmente (definido en base al intervalo intercuartílico), se añade el criterio de estabilidad.

La Figura 3 muestra la distribución de las respuestas en función de la existencia o no de consenso y estabilidad. Como se observa en ellas, en la mayoría de los elementos analizados se registra una estabilidad en las respuestas (variaciones inferiores al $50 \%$ en rango intercuartílico relativo). La única excepción es la orientación al mercado, en la que sí que se supera este valor, no obstante, aunque ello se traduce en las posturas de los expertos han variado, la lectura combinada junto con el elevado consenso que existe en relación a este factor permite señalar que es posible concluir las rondas de consulta puesto que o bien existe consenso o bien las respuesta obtenidas son estables. 


\section{Figura 3. Consenso y estabilidad en las respuestas en la segunda ronda (grado de coincidencia por parte de los expertos en la respuesta más frecuente y variación de las respuestas entre rondas)}

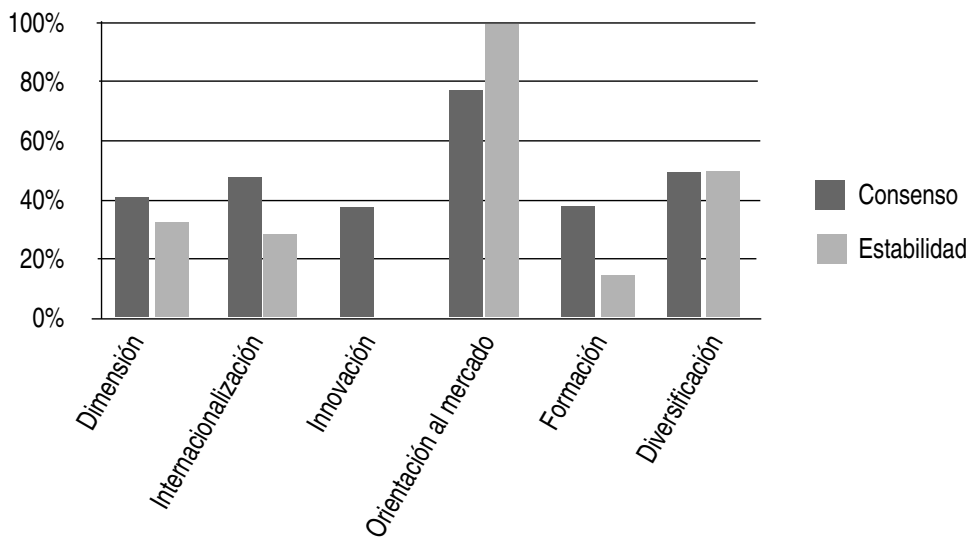

\section{6.- Discusión de resultados}

En la Tabla 1 se muestran los resultados obtenidos tras la consulta a expertos para la valoración sobre la importancia relativa de los factores de competitividad en cooperativas agroalimentarias españolas.

A pesar de que no ha sido posible encontrar una jerarquización que cuente con el consenso del panel de expertos, las respuestas obtenidas sí que permiten formular una propuesta de ordenación.

En ella tanto la orientación al mercado como la innovación ocupan la primera posición en relación a la importancia percibida. Esta percepción se corresponde con la opinión de diversos expertos que, en relación al conjunto de empresas en general, han señalado en los últimos años la relevancia de la orientación al mercado en la competitividad (Martín Armario, 2003; Vega Vázquez, 2004, entre otros) 0 de la innovación (Esteve y Rodríguez, 2014; Pulido y López, 2013; entre otros).

El ranking establece que les siguen en importancia la diversificación, la dimensión, la internacionalización y la formación. 


\section{Tabla 1. Valoración de los expertos sobre la importancia relativa de los factores de competitividad en cooperativas agroalimentarias}

\begin{tabular}{|l|ccccc|}
\hline & $\mathrm{m}$ & Media & Desv. estándar & ¿Existe consenso? & ¿Existe estabilidad? \\
\hline Dimensión & 3 & 2,85 & 1,46 & No & Sí \\
Internacionalización & 4 & 3,30 & 1,27 & No & Sí \\
Innovación & 1 & 2,35 & 1,41 & No & Sí \\
Orientación al mercado & 1 & 1,37 & 1,01 & Sí & No \\
Formación & 5 & 3,85 & 1,87 & No & Sí \\
Diversificación & 2 & 3,54 & 1,77 & Sí & Sí \\
\hline
\end{tabular}

FUENTE: Elaboración propia.

Adicionalmente, en el primer cuestionario remitido a los expertos, éstos contaban con la posibilidad de señalar factores de competitividad adicionales que en su opinión fueran relevantes en el caso particular de las cooperativas agroalimentarias.

Las respuestas recibidas no sugerían la necesidad de incluir en la segunda ronda del estudio nuevos factores respecto a la propuesta inicial, puesto que se trataban de cuestiones concretas de gestión que se podían englobar en algunos de los factores seleccionados.

Así, un elevado número de ellos estaban encaminados a procesos de integración (bien de cooperativas productoras, bien en redes de innovación o concentrando oferta), este aspecto lo consideramos incluido en el factor dimensión de la propuesta inicial.

Otro grupo de sugerencias estaban encaminadas a mejorar la comercialización del producto, incidiendo especialmente en la calidad de la red de comercialización, en el ajuste de producciones y demanda del mercado o en la promoción y diferenciación del producto. Estas cuestiones podrían quedar englobadas en la orientación al mercado.

Se aportó también la cuestión del grado de profesionalización en el sector, elemento que forma parte de la formación. Finalmente se señalaron también otros elementos como la calidad y la seguridad alimentaria, la estructura de costes o la intervención de la Administración Pública que realiza para dirigir estratégicamente al sector a través del uso de subvenciones. 


\section{7.- Conclusiones}

La competitividad es un elemento clave en cualquier organización a fin de asegurar la supervivencia en un entorno que resulta cada vez más hostil. Las estrategias empresariales se formulan a partir de análisis de posición a fin de explotar oportunidades o mejorar carencias.

Las cooperativas como organizaciones empresariales deben elaborar planes que les permitan mejorar su posición en el mercado.

Para mejorar la competitividad de las cooperativas es necesario que se trabaje específicamente en la formación, la internacionalización, la dimensión, la diversificación, la orientación al mercado y la innovación. La principal aportación de este trabajo es la propuesta de un orden de importancia de los anteriores elementos, obtenido a través de una consulta a expertos.

Tanto la orientación al mercado como la innovación son elementos que han sido propuestos por consenso como los más relevantes a la hora de incentivar la competitividad. Este resultado es coherente con la actual corriente de gestión en la que prima la necesidad de satisfacer no sólo al cliente sino al mercado. Además, en un sector como el agroalimentario en el que abundan las commodities, es vital buscar la diferenciación y una de las vías para lograrlo es la innovación.

Los siguientes factores en importancia son la dimensión, la internacionalización y la formación. Destaca particularmente este último aspecto, la formación, ya que pone de manifiesto uno de los problemas que tradicionalmente se ha achacado al sector y es la falta de profesionalización o de formación. Podemos afirmar que el hecho de que haya sido categorizado como el factor menos importante dentro de los propuestos denota en cierta forma que aún persiste esa mentalidad en el sector.

En futuras investigaciones sería interesante analizar si existen diferencias significativas entre los distintos grupos de expertos que han participado en el estudio, es decir si los gerentes de cooperativas tienen opiniones diferentes a las que proporcionan los investigadores o el personal de la Administración. Adicionalmente se podría profundizar estudiando si se podrían establecer dos tipos de factores: un grupo de elementos de carácter transversal que potencien el resto de aspectos (por ejemplo, la formación) y otro más específico, integrado por la innovación o la internacionalización. 


\section{7.- Coclusiones}

ALONSO RODRIGO, E. \& SANTA CRUZ AYO, I. (2016): "Identidad, competitividad y creación de empleo: retos para una nueva fiscalidad de las cooperativas de trabajo asociado", Boletín de la Asociación Internacional de Derecho Cooperativo, 50, 73-101.

ARCAS, N. (2002): "Contribución de las cooperativas agrarias de segundo grado a la orientación al mercado de sus cooperativas asociadas: efectos en el desempeño de la relación", CIRIEC-España, Revista de Economía Pública, Social y Cooperativa, 41, 139-161.

ARCAS-LARIO, N., MARTÍN-UGEDO, J.F. \& MÍNGUEZ-VERA, A. (2013): "Satisfacción de los socios con las cooperativas agrarias. Una explicación a partir de la Teoría de los Derechos de Propiedad", ITEA-Información Técnica Económica Agraria, 109(4), 443-457.

ARCAS-LARIO, N., MARTÍN-UGEDO, J.F. \& MÍNGUEZ-VERA, A. (2014): "Farmers' satisfaction with fresh fruit and vegetable marketing Spanish cooperatives: an explanation from Agency Theory", IFAMA- International Food and Agribusiness Management Review, 17(1), 127-146.

BENOS, T., KALOGERAS, N., VERHEES, F., SERGAKI, P. \& PENNINGS, J. (2015): “Cooperatives' organizational restructuring, strategic atributes and performance: the case of agribusiness cooperatives in Greece", Agribusiness, 32(1), 127-150.

BRETOS, I. \& MARCUELLO, C. (2017): "Revisiting globalization challenges and opportunities in the development of cooperatives", Annals of Public and Cooperative Economics, 88(1), 47-73.

CAMBRA, J.J. \& FUSTER, A. (2005): "Importancia de la medición del grado de orientación al mercado en el sector cooperativista como respuesta al nuevo marco competitivo", Investigaciones Europeas de Dirección y Economía de la Empresa, 11(3), 67-83.

CAMPOS-CLIMENT, V., APETREI, A. \& CHAVES-ÁVILA, R. (2012): "Delphi method applied to horticultural cooperatives", Management decision, 50(7), 1266-1284.

CAMPOS-CLIMENT, V. \& CHAVES-ÁVILA, R. (2012): "El papel de las cooperativas en la crisis agraria. Estudio empírico aplicado a la agricultura mediterránea española", Cuadernos de desarrollo rural, 9 (69), 175-194.

DELBECQ, A., VAN DE VEN, A., GUSTAFSON, D., VILLEGAS, C. \& Hernández, M. (1989): Técnicas grupales para la planeación, Ed. México Trillas. Citado en Gallego, L. y Julia, J.F. (2003): "Principios cooperativos y eficacia económica. Un análisis delphi en el contexto normativo español", CIRIECEspaña, Revista de Economía Pública, Social y Cooperativa, 44, 231-259. 
ENCINAS, B., CALATAYUD, E. \& GARCÍA, G. (2011): "Las cooperativas hortofrutícolas frente a la crisis. La necesaria apuesta por la competitividad. Aspectos económico-financieros", CIRIEC-España, Revista de Economía Pública, Social y Cooperativa, 72, 125-156.

ESTEVE, S. \& Rodríguez, D. (2014). La innovación como factor de competitividad de las PYMEs. Información Comercial Española, ICE: Revista de Economía, 877:115-130

FAYOS, T., CALDERON, H. \& MIR, J. (2011): "El éxito en la internacionalización de las cooperativas agroalimentarias españolas. Propuesta de un modelo de estudio desde la perspectiva del marketing internacional", CIRIEC-España, Revista de Economía Pública, Social y Cooperativa, 72, 43-72.

FERNANDEZ DE BOBADILLA, S. \& VELASCO, E. (2008): "Gestionan mejor la innovación las empresas de economía social que las sociedades anónimas: estudio comparativo de empresas innovadoras vascas", CIRIEC-España, Revista de Economía Pública, Social y Cooperativa, 63, 5-37.

GALLEGO, J.R. \& LAMANTHE, A. (2011): "¿Por qué las cooperativas no dominan en los sistemas hortofrutícolas tradicionales? Una comparación entre España y Francia", CIRIEC-España, Revista de Economía Pública, Social y Cooperativa, 72, 9-41.

GARCÍA-GUTIÉRREZ, C. (1988-1989): "El problema de la doble condición de los socios-trabajadores (socios-proveedores y socios-consumidores) ante la gerencia de la empresa cooperativa", REVESCO, Revista de Estudios Cooperativos, 56-57, 83-121.

GUZMÁN, I., D-NIEVES-NIETO, C. \& BRIONES-PENALVER, A.J. (2013): "Evaluación de la eficiencia en el sector de los agronegocios en España: un estudio empírico para la Región de Murcia", Cuadernos de Desarrollo Rural, 10(71), 81-100.

HERNÁNDEZ-ASCANIO, J., TIRADO-VALENCIA, P. \& ARIZA-MONTES, A. (2016): "El concepto de innovación social: ámbitos, definiciones y alcances teóricos", CIRIEC-España, Revista de Economía Pública, Social y Cooperativa, 88: 165-199.

HERNÁNDEZ-ESPALLARDO, M. \& ARCAS-LARIO, N. (2003): "The Effects of Authoritative Mechanisms of Coordination on Market Orientation in Asymmetrical Channel Partnerships", International Journal of Research in Marketing, 20(2), 133-152.

HERNÁNDEZ-ESPALLARDO, M., SÁNCHEZ-PÉREZ, M. \& SEGOVIA-LÓPEZ, C. (2011): "Exploitation and exploration-based innovations: The role of knowledge in inter-firm relationship with distributors", Technovation, 31, 203-215.

JULIÁ IGUAL, J.F. \& GALLEGO SEVILLA, L.P. (2003): "Principios cooperativos y eficacia económica. Un análisis Delphi en el contexto normativo español", CIRIEC-España, Revista de Economía Pública, Social y Cooperativa, 44, 231-259.

JULIÁ IGUAL, J.F., MELIÁ-MARTI, E. \& GARCÍA-MARTÍNEZ, G. (2012): "Strategies developed by leading EU agrifood cooperatives in their growth models", Service Business. An International Journal, $6(1), 27-46$. 
JULIÁ IGUAL, J.F., MELIÁ-MARTÍ, E. \& GARCÍA-MARTíNEZ, G. (2013): "Modelos y casos de éxito del cooperativismo agroalimentario en la Unión Europea en El papel del cooperativismo agroalimentario en la economía mundial", Mediterráneo Económico, 24, 139-154.

KYRIAKOPOULOS, K. (1998): "Agricultural cooperatives: organizing for market-orientation", AMA World Congress VIII, Punta del Este.

LAJARA-CAMILLERI, N. \& SERVER IZQUIERDO, R.J. (2016): "Orientación al mercado y tipología de las cooperativas agroalimentarias en base a la competitividad. Caso-estudio de las citrícolas españolas", REVESCO, Revista de estudios cooperativos, 121(2):145-172.

MAGRAMA, Ministerio de Agricultura, Alimentación y Medio Ambiente (2014): Anuario de Estadística.

MARTÍN ARMARIO, J. (2003): Orientación al mercado y proceso de internacionalización de empresas, Tesis doctoral.

MARTíNEZ PIÑEIRO, E. (2003): "La técnica del Delphi como estrategia de consulta a los implicados en la evaluación de programas", Revista de Investigación Educativa, 21 (2), 449-463.

MELIÁ, E. \& MARTÍNEZ, A. (2015): "Characterization and analysis of cooperative mergers and their results", Annals of Public and Cooperative Economics, 3 (86), 479-504.

MELIÁN, A. \& CAMPOS CLIMENT, V. (2009): "Emprendedurismo y Economía Social como mecanismos de inserción socio-laboral en tiempos de crisis", REVESCO, Revista de estudios cooperativos, 100, 43-67.

MELIÁN , A. y MILLÁN, G. (2007): "El cooperativismo vitivinícola en España. Un estudio exploratorio", REVESCO, Revista de estudios cooperativos, 93(3), 39-67.

MONTEGUT, Y. (2006): Análisis de los factores explicativos del éxito competitivo en las almazaras cooperativas catalanas, Tesis doctoral, Lleida.

MONZÓN, J.L. \& HERRERO, M. (2016): "Identificación y análisis de las características identitarias de la empresa social europea: aplicación a la realidad de los centros especiales de empleo de la economía española", CIRIEC-España, Revista de Economía Pública, Social y Cooperativa, 87, 295326.

MORAL-PAJARES, E., MOZAS-MORAL, A., BERNAL-JURADO, E. \& MEDINA-VIRUEL, M.J. (2015): "Efficiency and exports: Evidence from Southern European companies", Journal of Business Research, 68, 1506-1511.

MOYANO, J., PUIG, F. \& BRUQUE, S. (2008): "Los determinantes de la competitividad en cooperativas", CIRIEC-España, Revista de Economía Pública, Social y Cooperativa, 61, 233-249.

NILSSON, J., KIHLÉN, A. \& NORRELL, L. (2009). "Are traditional cooperatives an endangered species? About shrinking satisfaction, involvement and trust", International food and agribusiness management review, 12(4), 101-120. 
OSCAE, Observatorio Socioeconómico del Cooperativismo Agroalimentario Español (2013): http://www.agro-alimentarias.coop/cooperativismo_en_cifras

PERAZA, E., GÓMEZ-GARCÍA, J.M. \& ALEIXANDRE, G. (2016): "Los factores determinantes del comportamiento innovador de las cooperativas: un análisis para el caso de Castilla y León", REVESCO, Revista de estudios cooperativos, 122(3), 252-284.

PULIDO FERNÁNDEZ, J.I. \& LÓPEZ SÁNCHEZ, Y. (2013): La innovación como factor de competitividad turística. Estructura económica de los mercados turísticos, Ed. Síntesis.

RODRÍGUEZ, J. \& MOZAS, A. (2003): "La formación de los recursos humanos de las cooperativas agrarias y la nueva economía: un estudio empírico", Boletín económico del ICE, 2776, 9-19.

RUBIO-BAÑÓN, A. \& ARAGÓN-SÁNCHEZ, A. (2002): "Factores explicativos del éxito competitivo en la PYME", Cuadernos de gestión, 2 (1).

SAIZ, J.M. \& MENDOZA, M.M. (2015): "Innovación y creatividad como motores de desarrollo y RSC. El caso de las empresas de Guayaquil (Ecuador)", CIRIEC-España, Revista de Economía Pública, Social y Cooperativa, 85, 33-53.

TAMAYO, J.A., ROMERO, J.E., GAMERO, J. \& MARTÍNEZ-ROMÁN, J.A. (2015): "Do Innovation and Cooperation influence SMEs' competitiveness? Evidence from the Andalusian metal-mechanic sector", Innovar, Revista de Ciencias Administrativas y Sociales, 25(1), 101-115.

TRIGUERO-CANO, A. \& CUERVA, M.C. (2011): "El cooperativismo agroalimentario ante el reto de la globalización: renovarse o morir", CIRIEC-España, Revista de Economía Pública, Social y Cooperativa, 72, 97-124.

URDE, M., BAUMGARTH, C. \& MERRILEES, B. (2013): "Brand orientation and market orientation From alternatives to synergy", Journal of Business Research, 66, 13-20.

VEGA VÁZQUEZ, M. (2004): Orientación al mercado e innovación en nuevos productos: Antecedentes organizativos, Tesis doctoral.

VEZINA, M., MALO, M.C. \& BEN SELMA, M. (2017): "Mature Social Economy Enterprise and Social Innovation: the case of the Desjardins Environment Fund", Annals of Public and Cooperative Economics, 88(2), 257-278. 
